\title{
Aspectos técnicos da esofagocardiomi otomia com divulsão para o tratamento cirúrgico do megaesôfago chagásico não avançado
}

\author{
B. Pilon, F.V. Teixeira, J .P.I. Terrazas, E.P. Moreira, E.Y. Pillon
}

Departamento de Cirurgia, Disciplina de Cirurgia do Aparel ho Digestivo, Hospital de Clínicas II, Faculdade de Medicina de Marília, Marília, SP.

RESUMO - ОвJ ETIVo. Os autores descrevem uma variante da técnica proposta por Heller para o tratamento cirúrgico do megaesofago: esofagocardiomiotomia com divulsão associada a esofagofundogastropexia.

Casuística e Método. No período de junho de 1988 a março de 1996, foram operados, na Disciplina de Cirurgia do Aparelho Digestivo do Departamento de Cirurgia da Faculdade de Medicina de Marília, SP, 50 pacientes portadores de megaêsofago chagásico graus I, II e III.

Resultados. Num seguimento de seis meses a 7,6 anos, os resultados são classificados como ótimo,

\section{INTRODUÇÃO}

O megaesôfago chagásico (MC) é uma doença importante no Brasil por causa da sua alta prevalência e quadro clínico grave de desnutrição, levando até a caquexia ${ }^{1}$. A pós ter sido conhecida a fisiopatologia da doença, acalásia do esfíncter inferior do esôfago (EIE), a cirurgia é, de longe, o procedimento terapêutico com melhores resultados, quando comparada à dilatação com balão ${ }^{2-4}$. A destruição do EIE, por meio da esofagocardiomiectomia (MIEC) ou da esofagocardiomiotomia (MIO), promove a melhora da disfagia na quase totalidade dos casos de MC incipiente e MC nãoavançado ${ }^{5-7}$, reservando o tratamento dilatador para casos selecionados de pacientes sem condições cirúrgicas por causa do elevado grau de desnutrição $0^{7,8}$.

O objetivo deste trabalho é apresentar uma modificação na técnica operatória utilizada para o tratamento cirúrgico do MC, graus I, II e III (classificação de Ferreira Santos) ${ }^{8}$, que consiste numa variante da cirurgia inicialmente proposta por Heller e, posteriormente, modificada por Mattos $^{9,10}$.

Como alternativa para a MIEC ou para MIO, é realizada uma esofagocardiomiotomia com divulsão (MIOD) da musculatura esofagogástrica e, como procedimento anti-refluxo, é efetuada uma
$86 \%$, e bom, $14 \%$. O estudo radiológico contrastado mostra melhora do clareamento esofágico e a endoscopia, ausência de restos alimentares e esofagite.

Conclusão. Os autores concluem que esofagocardiomiotomia com divulsão associada a esofagofundogastropexia é eficaz no tratamento cirúrgi co do megaesôfago chagási co graus I, II e III, e enfatizam a facilidade técnica e a segurança do procedimento.

Unitermos: Doença de Chagas. Acalásia. Cirurgia.

esofagofundogastropexia semelhante à descrita por Pinotti et al. ${ }^{11}$

Enfatizamos os excelentes resultados obtidos em 50 pacientes, analisando parâmetros clínicos, cirúrgicos, radiológicos e endoscópicos.

\section{CASUÍSTICA E MÉTOdOS}

No período de junho de 1988 a março de 1996, foram operados, na Disciplina de Cirurgia do Aparelho Digestivo do Departamento de Cirurgia da Faculdade de Medicina de Marília e hospital privado, 50 paci entes portadores de MC, sem predominância por sexo, com idade que variou de 20 a 74 anos, média de 44 anos, com prevalência maior na raça branca (86\%), em relação à negra.

Todos os pacientes tinham quadro clínico de disfagia cujo intervalo do sintoma variou de seis meses a 20 anos (média de 10,2 anos). Vinte e sete (54\%) queixaram-se de odinofagia e 26 (52\%) relataram emagrecimento, com perda ponderal média de $12 \mathrm{~kg}$. Dezesseis pacientes (32\%) eram portadores de problemas respiratórios, entre os principais a broncopneumonia e a "sufocação noturna" (tabela 1).

O diagnóstico foi feito através de história clínica, exame radiológico contrastado ${ }^{8}$ e endoscopia digestiva. 


\begin{tabular}{|lcr|}
\hline \multicolumn{2}{|c|}{ Tabela 1 - Sintomas iniciais - Pré-operatório } \\
\hline Sintomas & $N^{\circ}$ pacientes & $\%$ \\
Disfagia & 50 & 100 \\
Odinofagia & 27 & 54 \\
Emagrecimento & 26 & 52 \\
Pneumonia de repetição & 16 & 32 \\
\hline
\end{tabular}

Tabela 2 - Grau do megaesôfago chagásico

\begin{tabular}{ccc}
\hline Grau do megaesôfago & №pacientes & $\%$ \\
Grau I & 2 & 4 \\
Grau II & 30 & 60 \\
Grau III & 18 & 36
\end{tabular}

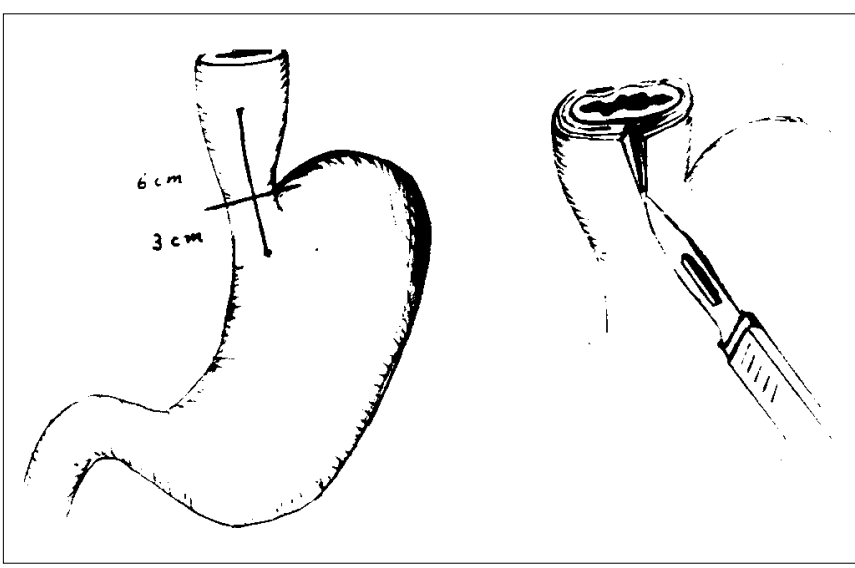

Fig. 1 - Esofagocardiomi otomia de $9 \mathrm{~cm}$ de extensão.

F oi utilizada a classificação radiológi ca proposta por Ferreira Santos, em 1963. Após ingestão de $100 \mathrm{~g}$ de bário e $200 \mathrm{~mL}$ de água, foram obtidas três chapas (exposições) em tempos diferentes (10", 5' e 30 '), com o paciente em pé, colocado a uma distância padrão do filme de 180 centímetros. Dois (4\%) pacientes eram portadores de MC grau I, 30 (60\%) grau II e 18 (36\%) grau III (tabela 2). Todos pacientes apresentavam dificuldade de clareamento esofágico, caracterizado pela presença de contraste nas chapas de 10 segundos, 5 e 30 minutos. A ausência da bolha gástrica foi vista em $88 \%$ (44/50). Nos MC grau II e III (96\%), a endoscopia digestiva revelou aumento do lume esofágico e a presença de restos alimentares.

Todos os pacientes foram submetidos a MIOD associada a esofagofundogastropexia.

\section{Técnica operatória}

1) Incisão mediana supra-umbilical;

2) I solamento do esôfago abdominal e reparo com dreno de penrose;

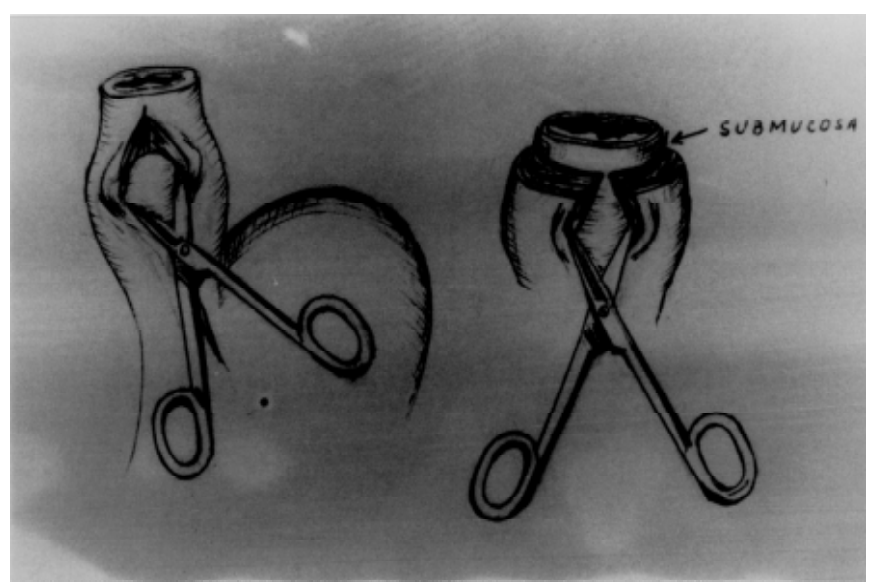

Fig. 2 - Divulsão com tesoura da musculatura circular e longitudinal do esôfago.

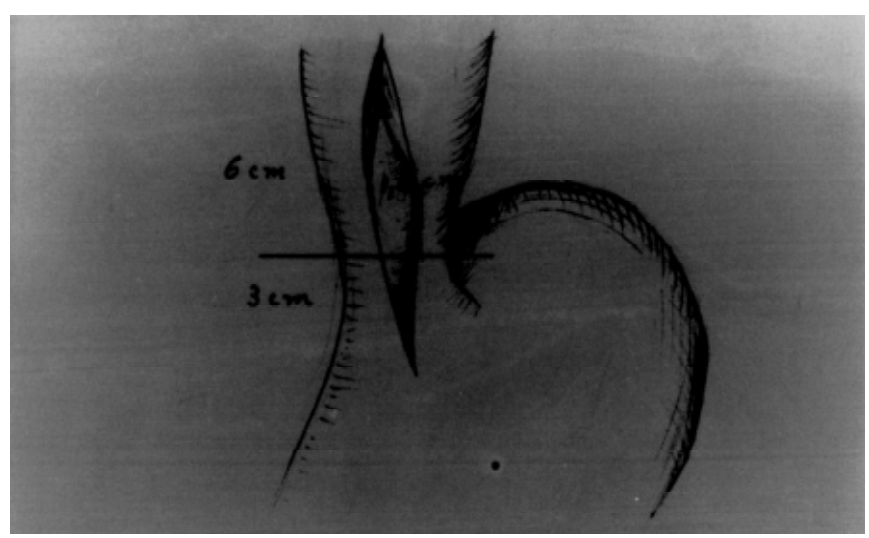

Fig. 3 - Bordas da miotomia afastadas em $1,2 \mathrm{~cm}$. Nítida herniação da submucosa esofágica e gástrica.

3) I sol amento e cadarçamento do vago anterior;

4) Ligadura dos vasos situados anteriormente à cárdia com fio absorvível;

5) Miotomia de $9 \mathrm{~cm}$ de extensão, sendo $6 \mathrm{~cm}$ no esôfago passando pela cárdia e $3 \mathrm{~cm}$ no estômago, como preconizou Heller ${ }^{9}$ (fig. 1);

6) Divulsão, com tesoura, da musculatura seccionada, e nos casos em que a submucosa se encontra aderida às camadas musculares (esofagite), dissecção cuidadosa e rotura dos feixes musculares remanescentes com gaze seca presa a uma pinça hemostática (fig. 2);

7) Ao longo de toda extensão da miotomia $(9 \mathrm{~cm})$, observa-se uma área exposta entre as bordas que varia de 1,2 a 1,6cm de largura. A visibilização de bol has de ar movendo-se na luz esofagogástrica e a herniação completa da submucosa asseguram o sucesso da miotomia (fig. 3);

8) Confecção de uma válvula anti-refluxo (esofagofundogastropexia) recobrindo a área exposta de submucosa com duas suturas entre a parede anterior do fundo gástrico e as bordas da miotomia, e 


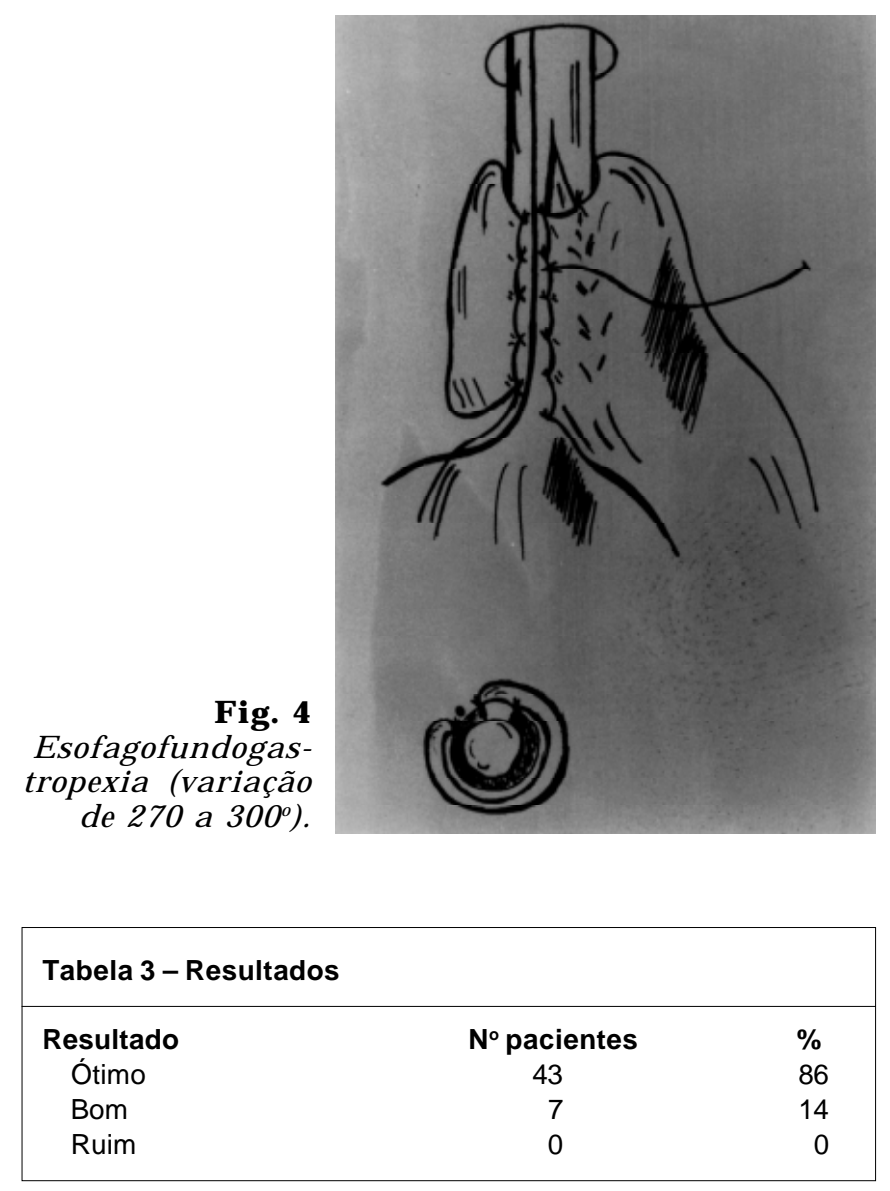

Tabela 4 - Resultado - Sintomas pré e pós-operatórios

\begin{tabular}{|lcc|}
\hline \multicolumn{1}{c}{ Sintomas } & $\begin{array}{c}\text { \% Sintomas } \\
\text { Pré-op }\end{array}$ & $\begin{array}{c}\text { \% Sintomas } \\
\text { Pós-op }\end{array}$ \\
Disfagia & 100 & 0 \\
Odinofagia & 54 & 0 \\
Emagrecimento/ ganho de peso & 52 & 62 \\
Pneumonia de repetição & 0 & 0 \\
\hline
\end{tabular}

outra sutura entre a parede anterior do fundo gástrico e a parede póstero-lateral direita do esôfago ${ }^{11}$. Foram utilizados fios inabsorvíveis 2.0 (fig. 4).

\section{RESULTADOS}

Para a avaliação dos resultados, foram utilizados critérios clínicos, radiológicos e endoscópicos.

Numa tentaiva de padronizar nossos resultados, estabelecemos, previamente, critérios clínicos para uma posterior avaliação․ $\mathrm{Na}$ avaliação clínica, consideramos como resultado ótimo aqueles pacientes que, após a operação, ti veram regressão completa dos sintomas, principalmente a disfagia. Bom, aqueles com disfagia esporádica para sólidos e ausência de sintomas decorrentes de

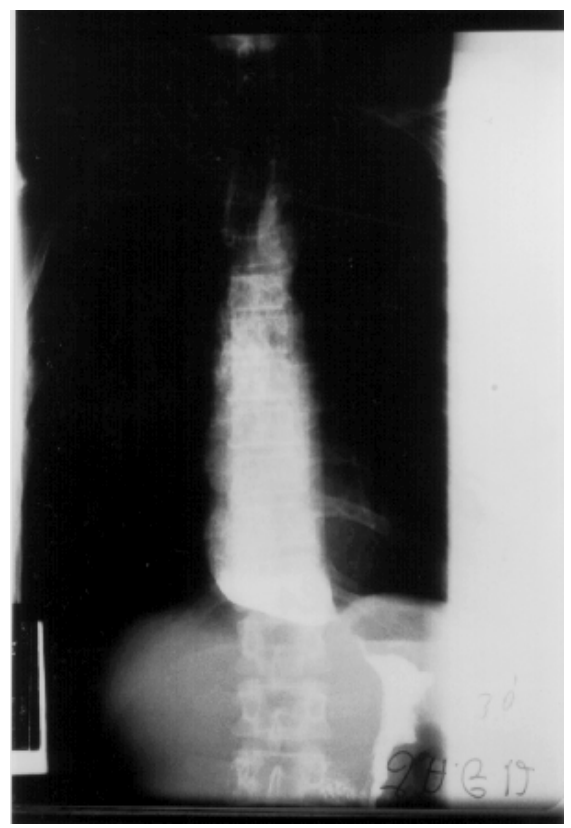

Fig 5A

E sofagograma com técnica padrão $180 \mathrm{~cm}$; antes da miotomia. Chapa de 30 minutos mostra estase do material baritado e dilatação do esôfago.

refluxo gastroesofágico. Ruim, os que permaneceram com disfagia para sólidos, líquidos e/ou sintomas de refluxo.

Tivemos, em nossa casuística, os seguintes resultados: Ótimo, 86\% (43/50); bom, 14\% (7/50) (tabela 3).

E $m$ todos os portadores de odinofagia e problemas respiratórios, houve desaparecimento completo dos sintomas.

Trinta e um pacientes (62\%) apresentaram ganho de peso que variou de 1 a $20 \mathrm{~kg}$ (tabela 4).

$\mathrm{Na}$ avaliação radiológica, constatou-se diminuição na col una de bário em torno de 50 e $80 \%$ aos 10 segundos e 5 minutos, respectivamente, e ausência total de bário na chapa de 30 mi nutos. Diminuição no diâmetro do esôfago e aparecimento da bolha gástrica foram observados em todos casos (figs. 5A e 5B).

A endoscopia não visibilizou, em nenhum caso, a presença de restos alimentares ou de lesão compatível com esofagite.

Todos os pacientes receberam avaliação periódica após a cirurgia, que variou de seis meses a 7,6 anos, inicialmente mensal e, posteriormente, semestral. Durante 12 meses, foram realizados estudos endoscópicos e radiológicos em retornos semestrais. Decorrido esse período, foi realizado acompanhamento anual, com avaliação clínica, radiológica e endoscópica.

Não houve nenhum óbito nessa casuística. Tivemos dois (4\%) casos de perfuração esofágica, durante o ato operatório, que foram solucionados com ráfia da lesão (pontos simples, inabsorvíveis mo- 


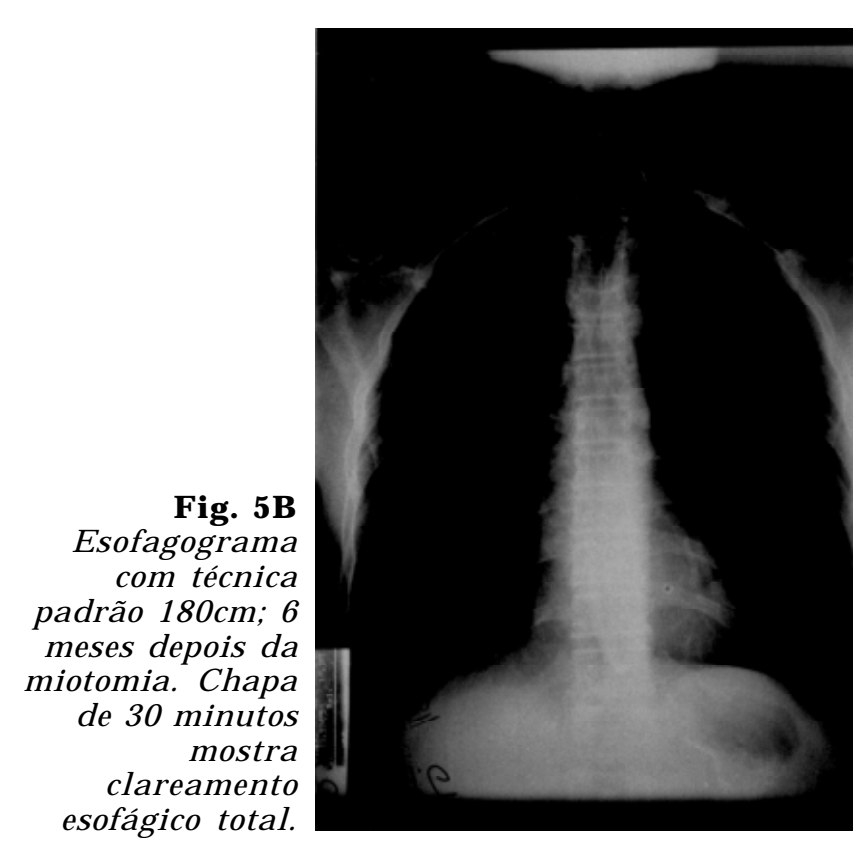

nofilamentares). Esses pacientes evoluíram bem, sem intercorrências. Foram constatados doi s casos de broncopneumonia, no pós-operatório imediato, que tiveram melhora do quadro com tratamento clínico com antibióti cos e fisioterapia respi ratória. Dois pacientes apresentaram hérnia incisional no pós-operatório tardio, que foram sol ucionadas com o tratamento cirúrgico (herniorrafia).

\section{DISCUSSÃo}

A proposta de modificar a técnica cirúrgica idealizada por $\mathrm{Heller}^{9}$ teve como objetivo principal a busca de um procedimento que acreditávamos ser de maior facilidade e segurança, quando comparado à MIEC.

A real ização da mi otomi a esofágica numa extensão de $6 \mathrm{~cm}$, passando pela cárdia e estendendo-se até $3 \mathrm{~cm}$ sobre o estômago, promove a destruição do EIE, facilmente identificado por se apresentar como uma área de afilamento na extremidade distal do esôfago, junto à transição gástrica.

A divulsão das camadas musculares do esôfago com tesoura faz com que a musculatura, fácil e seguramente, se separe da submucosa e deslize num verdadeiro plano de clivagem, sem risco ou com risco mínimo de perfuração. As bordas da miotomia se afastam numa distância que pode variar de 1,2 a $1,6 \mathrm{~cm}$ ou mais. A divulsão da parede muscular do esôfago e do estômago proporciona uma herniação completa da submucosa. Tal herniação, associada a visibilização de bolhas de ar movendo-se na luz esofágica, ratifica o sucesso da cirurgia.
Entretanto, quando existe esofagite importante, como acontece nos casos de estase significativa, principalmente no MC grau III, a musculatura da parede esofágica adere à submucosa e não se sol ta com facilidade, dificultando a divulsão e aumentando o risco de perfuração devido à presença de feixes musculares aderidos à submucosa, principalmente nos locais de penetração vascular.

Durante o desenvolvimento da técnica, deparamo-nos com esse problema que ocasionou a perfuração esofági ca em dois casos (4\%). N esses casos, deve-se fazer uma verdadeira dissecção, com o máximo de cautela, com tesoura e "turunda" (gaze seca presa a uma pinça hemostática). Caso venha a ocorrer perfuração esofágica, esta é de diâmetro pequeno, facilmente identificada e reparada com sutura simples.

São descritos excelentes resultados para o tratamento cirúrgico do MC não-avançado, com a técnica da MIEC associada a esofagofundogastropexia, sem recidiva da disfagia ${ }^{3-6}$. Entretanto, alguns autores apontam índices de recorrência da disfagia que variam de 8 a 17,1 \% $\%^{7,12}$.

Além da indicação incorreta da cirurgia e da estenose esofágica causada pela não-realização de um procedimento anti-refluxo e da fibrose da miotomia (17\%), a mi otomia incompleta (49\%) é a principal causa de insucesso do tratamento cirúrgico do $\mathrm{MC}^{13-15}$.

O fato de não termos tido casos de recidiva de disfagia, em nossa casuística, pode ser explicado pel o efetivo distanciamento das margens da miotomia consegui do através da divulsão da musculatura, associada a sutura da parede gástrica nas bordas da incisão. Contudo, é descrito, na literatura, que a realização da esofagomiotomia sem divulsão da parede muscular proporciona afastamento adequado das bordas, fato este não observado em outros pacientes, portadores de MC, anteriormente operados por nós e que motivou a realização da MIOD ${ }^{16,17}$.

A perfuração esofágica, complicação com incidência de até $19 \%$, é importante fator que leva a maior morbidade e mortalidade operatória na MIEC ${ }^{7,12}$. Talvez, em alguns estudos, a causa desse el evado grau de perfuração seja decorrente de uma maior dificuldade técnica para a retirada da fita muscular, quando comparada a miotomia, principalmente nos casos de MC acompanhados de esofagite.

Os resultados clínicos obtidos e classificados como ótimo (86\%) e bom (14\%), com relação a disfagia e sintomas de refluxo, ganho significativo de peso, desaparecimento da odinofagia e meIhora dos problemas respiratórios, podem ser jus- 
tificados pelas mudanças radiológicas e endoscópicas que caracterizaram efetiva melhora do clareamento esofágico do material baritado, diminuição do calibre do esôfago, presença da bol ha gástrica e ausência de restos alimentares ou sinais de esofagite.

Todos esses elementos comprovam a indubitável e perene destruição do EIE, o efetivo afastamento das bordas da miotomia, a provável participação da musculatura local mantida e a eficiência da válvula anti-refluxo.

Nossa avaliação quanto à eficácia da MIOD é baseada na clínica que evidencia o desaparecimento da disfagia, no estudo radiológico que mostra melhoria do clareamento esofágico e na endoscopia que não constata presença de restos alimentares e esofagite. Não pudemos contar com o auxílio da manometria esofágica, que, apesar de não ser indispensável para o diagnóstico do MC, é um exame imprescindível para comprovarmos a destruição do EIE. O exame pressórico do órgão poderia confirmar a diminuição das pressões basais do corpo esofágico e do EIE $^{17,18}$. Contudo, esse exame nem sempre está disponível, como foi em nosso caso e que deve ocorrer em outros lugares. No entanto é possível, em $100 \%$ dos casos - através da história clínica, de exames radiológicos contrastados e endoscopia -, fazer o diagnóstico do MC e comprovar a eficácia terapêutica do procedimento cirúrgico adotado ${ }^{1,17}$.

Condescendemo-nos com aquel es que acreditam "que, independente do valor dos procedimentos adotados para investigação dos resultados morfológi cos e funcionais e da sua importância para se compreender mel hor a fisiopatologia do MC, a instituição de critérios clínicos, adequadamente padronizados e interpretados, também nos oferece subsídios importantes para aquilatar os resultados pretendidos"1.

A MIOD associada a esofagofundogastropexia é um procedimento cirúrgico que pode ser realizado em centros menores por cirurgiões familiarizados com operações do esôfago, com riscos mínimos de perfuração esofágica e com ausência de recorrência da disfagia.

\section{CONCLUSÃO}

Concluímos que a esofagocardiomiotomia com divulsão associada a esofagofundogastropexia é um procedimento que pode ser usado, com excelente resultado, no tratamento cirúrgico do megaesôfago chagásico não-avançado, além de ser um procedimento fácil e seguro.

\section{AGRADECIMENTOS}

Os autores agradecem aos Drs. Massayoshi Tan e Eduardo Lemos de Souza Bastos, pelos desenhos da técnica operatória, e ao Sr. Carlos Fernandes dos Santos, pelas fotografias.

\section{SUMMARY}

Technical aspects of esophagomyotomy with divulsion for early chagasic megaesophagus surgical treatment

BACKgRound. The authors describe a Heller's technique alteration used for treatment of early Chagasic megaesphagus (ECM): esophagocardiomyotomy with divulsion plus esophagocardiopexy.

Patients and Methods. Between J une 1988 and March 1996, fifty patients were operated on at Surgery Department of FAMEMA. All had chagasic megaesophagus degrees I, II and III.

RESULTS. The results were excel lent in $86 \%$ (43/ 50 ) and good in $14 \%$ (7/50), for 6 months to 7.6 years of follow up. The radiological and endoscopic studies showed neither esophagic stasis nor food residues and esophagitis.

Conclusion. The authors concluded that esophagocardiomyotomy with divulsion plus esophagocardiogastropexy is efficient in ECM degrees I, II e III and emphasize both technical facility and security. [Rev Ass Med Brasil 1998; 44(3): 179-84.]

KEY WORDS: Chagas disease. Achalasia. Surgery.

\section{REFERÊNCIAS BIBLIOGRÁFICAS}

1. Barbosa H, BarichelloAW, Vianna AL, Mendelssonh P, Souza J AG. M egaesôfago chagási co: tratamento pel a cardioplastia à Thal. Rev Col Bras Cir 1981; 7(1): 16-29.

2. Ellenbogen G, Ishioka S, Pinotti HW, Raia A. Resultado do tratamento cirúrgico do megaesôfago não avançado. Aspectos morfológicos do esôfago operado. Rev Paul Med 1982; 99: 12-6.

3. Cecconello I. Tratamento do megaesôfago chagásico. Rev Ass Med Brasil 1984; 30: 1.

4. Andreollo NA, Brandalise NA, Leonerdi LS. Megaesôfago incipiente. Dilatação ou cirurgia? Rev Ass Med Brasil 1984; 30 : 4-6.

5. Pinotti HW, Habr-Gama A, Cecconellol, Felix VN, Zilbertein. The surgical treatment of megaesophagus and megacolon. B Dig Dis 1993; 11: 206-15.

6. Pinotti HW, Cecconello I, Zilbertein B. Megaesôfago. In Pinotti HW, Cecconello I, Gama-Rodrigues J , Habr-Gama A, Machado MCC, Saad WA, FaintuchJ (eds). Tratadodeclínica cirúrgica do aparel ho digestivo. 1aㅡ ed. São Paulo, Atheneu, 1994; 316-45.

7. Henry MACA, Spadella T, Leite CVS, Saad LHC. Megaesôfago: análise dos resultados detratamentocirúrgico XXXII Congresso Brasil ei ro de Gastroenterol ogia. Natal, 1992.

8. Ferreira-Santos R. Tratamento cirúrgi co da aperistalse esofágica (megaesôfago). Análisecrítica da experiência do Depar- 
PILON, B et al.

tamento de Cirurgia da Faculdade de Medicina de Ribeirão Preto. Tese, Ribeirão Preto, São Paulo, 1963.

9. Heller E. Extramukose Cardioplastik bein chronischen cardiospasmus mit dilatation des oesophagus. Mitt Grenzgeb M ed Chir 1913; 27: 141.

10. Mattos J O. Tratamento de megaesôfago pela esfincterotomia cárdica. Rev Ass Paul Med 1938; 4: 217.

11. Pinotti HW, Gama-Rodrigues J, Ellenbogen G. Nova técnica no tratamento cirúrgico do megaesôfago. Esofagocardiomiotomia associada com esofagofundogastropexia. Rev Goiana Med 1974; 20: 1-3.

12. Aquino J LB, I shida PCF, Reis Neto J F, Aquino P. Ressecção imediata na perfuração esofágica. XXXII Congresso Brasileiro de Gastroenterol ogia. Natal, 1992.

13. Pinotti HW, Felix VN, Domene CE, Purceli EL, Raia A. Recorrência da disfagia em pacientes operados de megaesôfago: análise dos fatores determinantes. Rev Ass Med Brasil 1980; 26: 109-14.

14. Pinotti, HW, Cecconello I, Felix VN, Sallum RA, Rocha J M. Reintervenção cirúrgica na recidiva da disfagia, após mioto- mia inadequada, no megaesôfago. VI Congresso Nacional do Colégio Brasileiro de Cirurgia Digestiva. Porto Alegre, 1994.

15. Cecconello I, Carvalho P, Nasi A, Felix VN, Pinotti HW. Fisiopatologia da recidiva da disfagia após cardiomiectomia. VI Congresso Nacional do Colégio Brasileiro de Cirurgia Digestiva. Porto Alegre, 1994.

16. Chaib SA. Tratamento cirúrgico do megaesôfago, uma nova modificação da operação de Heller. Rev Col Bras Cir 1983; 5: 169-73.

17. Ximenes Netto $M$, Marra O, Debiase $H$ et al. Primary surgical treatment of Chaga's disease megaesophagus. Results of 450 cases. HFA - Publ. Téc. Cient. Brasília 1987; 2(2): 147-61.

18. Ellenbogen G, Gama-Rodrigues J J , Betarello A, Pinotti HW, Raia A. Resultados do tratamento cirúrgico do megaesôfago chagásico não avançado. Aspectos funcionais do esôfago operado. Rev Hosp Clin Fac Med S Paulo 1981; 36: 252-8.

19. Teixeira FV, Pilon B, Terrazas J PI, Pillon EY. Tratamento cirúrgico do megaesôfago chagásico avançado recidivado em paciente gastrectomizado. Retificação esofágica. U ma alternativa. Rev Bras Cir 1997; 87(3): 113-8. 